\title{
Supporting Information: Stochastic Spiking Behavior in Neuromorphic Networks Enables True Random Number Generation
}

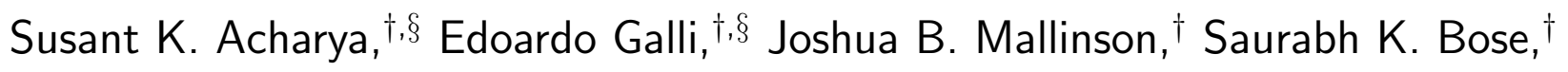
Ford Wagner, ${ }^{\dagger}$ Zachary E. Heywood, ${ }^{\ddagger}$ Philip J. Bones, ${ }^{\ddagger}$ Matthew D. Arnold,, and Simon A. Brown ${ }^{*, \dagger}$

$\dagger$ The MacDiarmid Institute for Advanced Materials and Nanotechnology, School of Physical and Chemical Sciences, Te Kura Matū, University of Canterbury, Private Bag 4800,

Christchurch 8140, New Zealand

$\ddagger$ Electrical and Computer Engineering, University of Canterbury, Private Bag 4800,

Christchurch 8140, New Zealand

ISchool of Mathematical and Physical Sciences, University of Technology Sydney, PO Box

123 Broadway NSW 2007, Australia

$\S S . K . A$. and E.G. contributed equally to this paper.

E-mail: simon.brown@canterbury.ac.nz 


\section{Supporting Information}

a

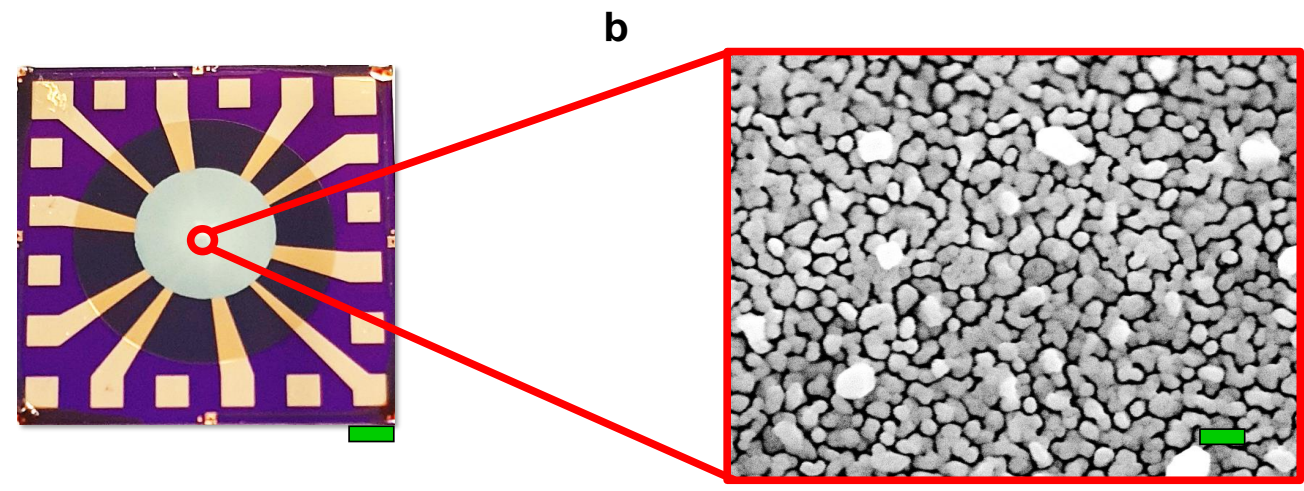

Figure S1: Images of a typical multi-contact PNN device. (a) Optical image of whole device. Scale bar: $1.2 \mathrm{~mm}$. (b) High magnification SEM image of the network of nanoparticles. Scale bar: $200 \mathrm{~nm}$. 

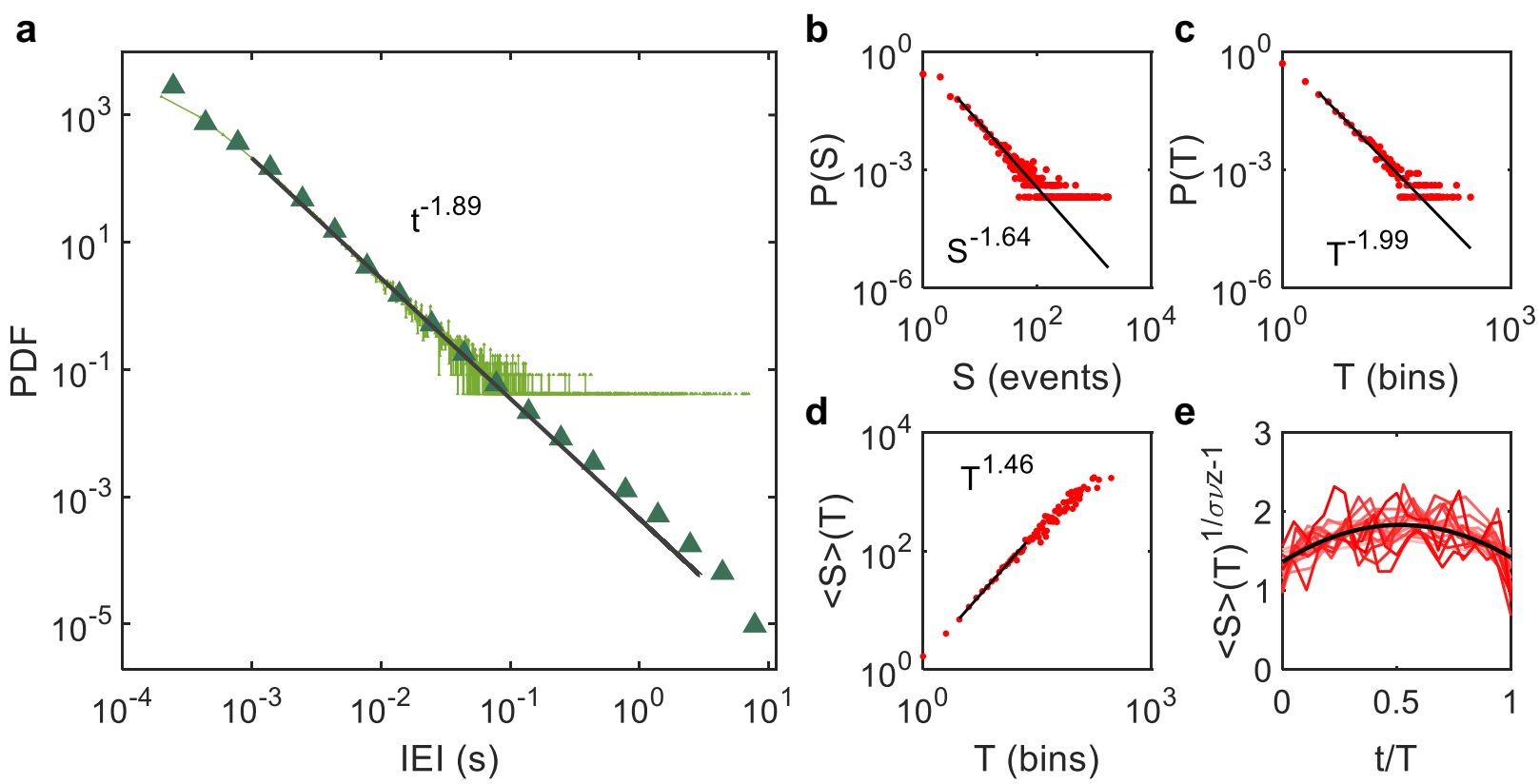

Figure S2: Inter-event intervals, and avalanche and criticality analyses. The data presented here are all consistent with previous work, demonstrating that criticality (see ${ }^{1}$ for details) is unaffected by differences in measurement technology and in device geometry (see main text and Methods). For this analysis the data shown in Figure 2a, obtained with a sampling interval of $10 \mu \mathrm{s}$, is sub-sampled at $200 \mu$ s intervals to be consistent with Refs. $1-3$. (a) The probability density function (PDF) of the inter event intervals follows a power law with a slope $\sim 1.89$, similar to that reported in previous work. ${ }^{2}$ Power law behavior is associated with correlated avalanches. ${ }^{1,2}$ Light and dark green: PDFs obtained using linear and logarithmic bin sizes for all IEIs, respectively. The power law maximum likelihood (ML) fit is shown as the black line. (b-d) Sizes $(\mathrm{S})$ and durations $(\mathrm{T})$ of the avalanches as well as the average avalanche size for given duration $<\mathrm{S}>(\mathrm{T})$ are distributed as power laws. (e) The avalanche shape collapse is comparable to those previously reported in Refs. 1,3 and in cortical tissue. ${ }^{4}$ The critical exponent $1 / \sigma \nu z$ is obtained from the crackling relationship $(\alpha-1) /(\tau-1)$, mean avalanche size given duration $<\mathrm{S}>(\mathrm{T})$, and avalanche shape collapse was found to be $1.55 \pm 0.2,1.46 \pm 0.02$, and $1.41 \pm 0.03$, respectively. [Uncertainties are one standard deviation.] The three independent estimates of $1 / \sigma \nu z$ agree with each other and therefore they fulfill the rigorous requirement for criticality. 


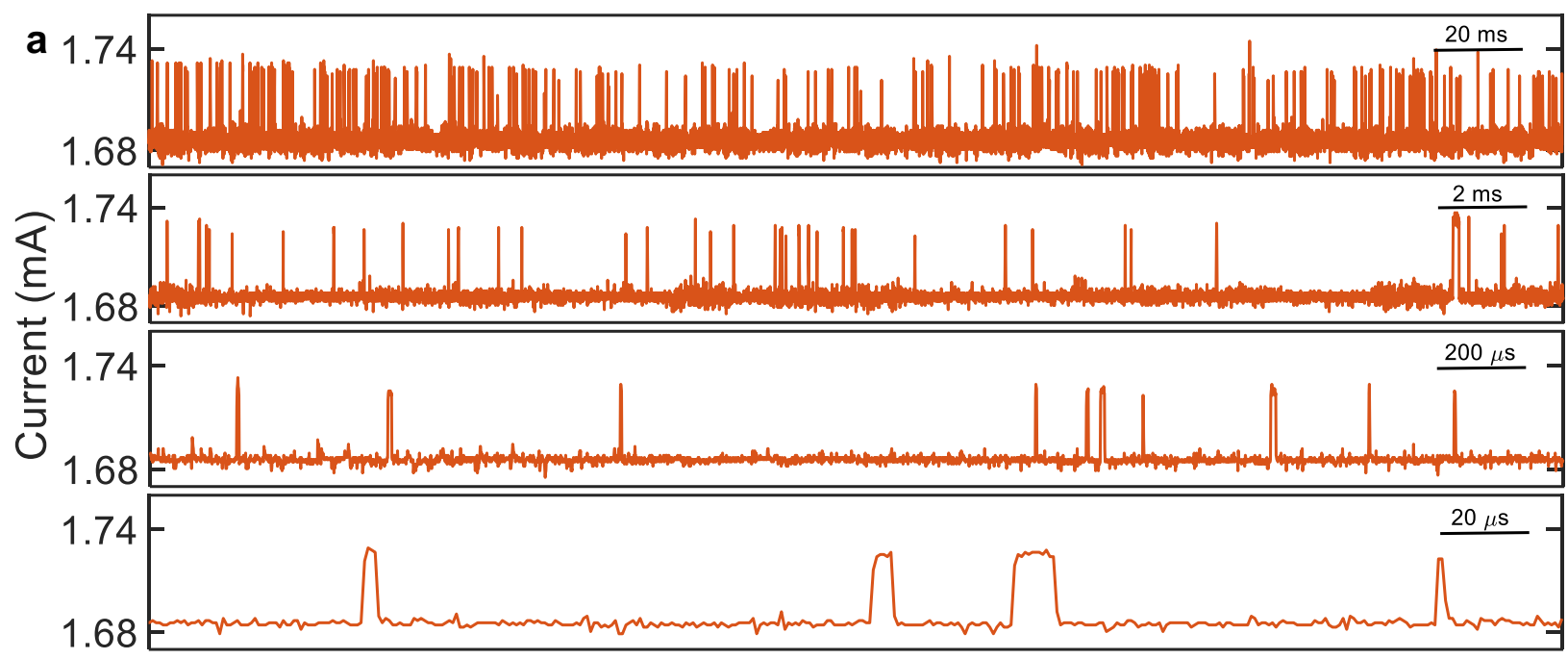

Time

b

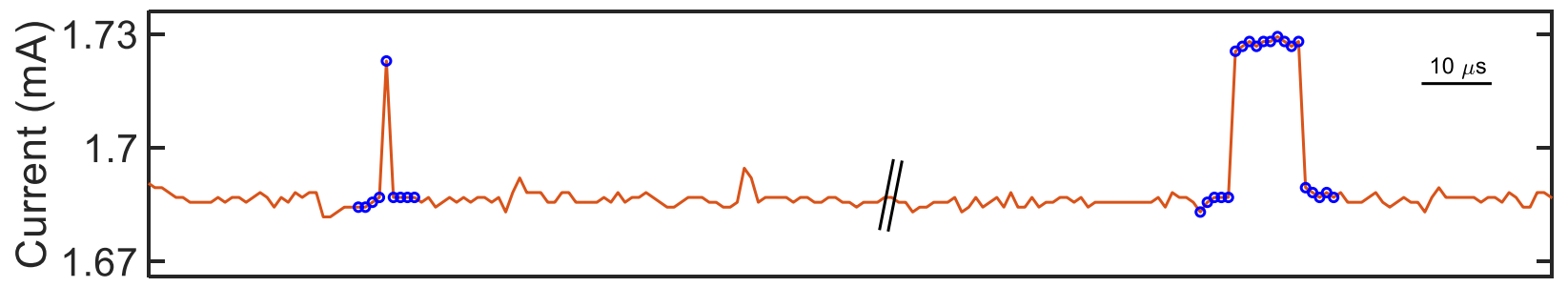

Time

Figure S3: Examples of spiking activity $\left(t_{U}<<t_{D}\right)$ measured with $1 \mu$ s sampling interval. Spiking is due to repeated switching events at a single site in the network. (a) Segment of data measured from Device 4. Each panel shows data at 10 times shorter time scale than the preceding one. The fourth panel shows that each spike is fully resolved with times in the UP state longer than $1 \mu \mathrm{s}$. (b) Further zoomed in view of two spikes from panel a showing that the transition between states (switching time) can be faster than $1 \mu \mathrm{s}$. $t_{U}$ for the spike on the left is $\sim 1 \mu \mathrm{s}$ while that for the spike on the right is $\sim 10 \mu \mathrm{s}$. Unresolved spikes occur only rarely at $1 \mu$ s sampling intervals (see Figure S9). 


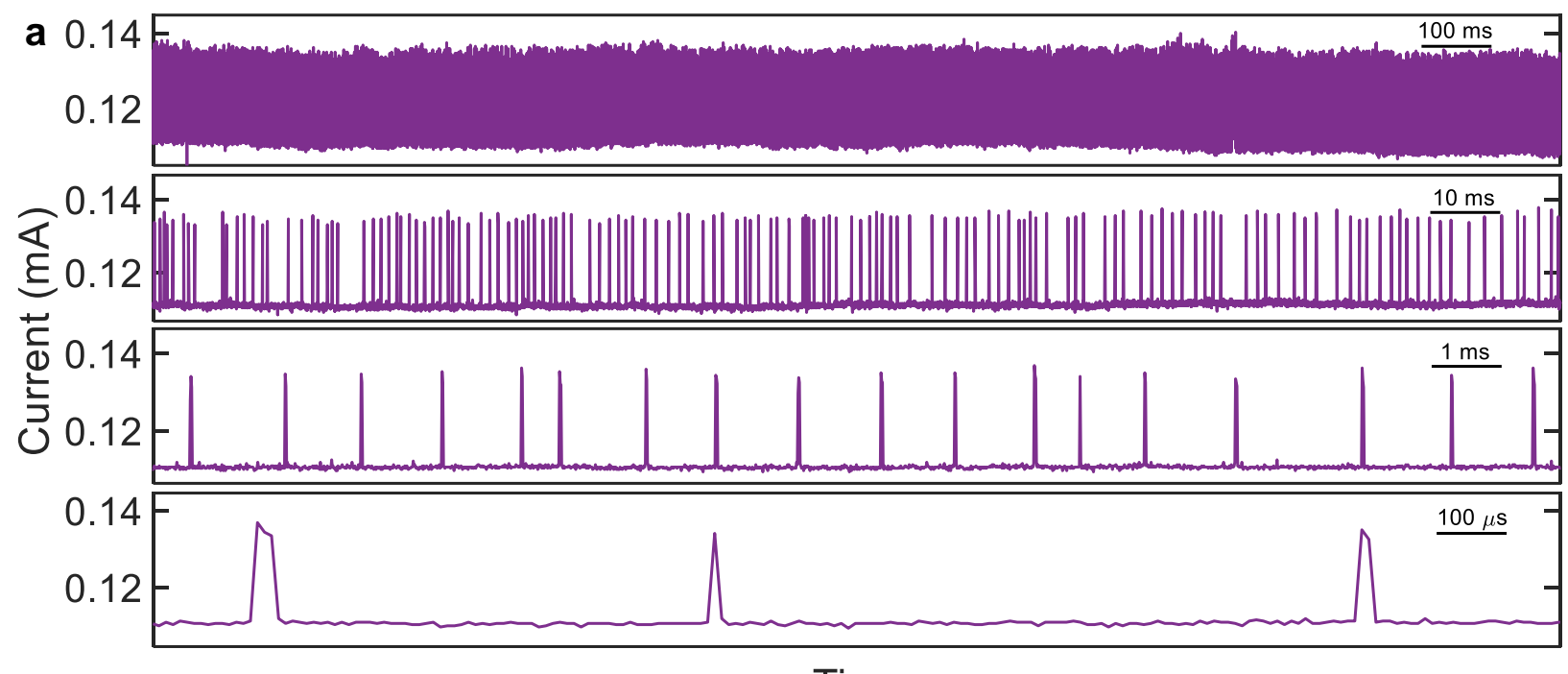

Time

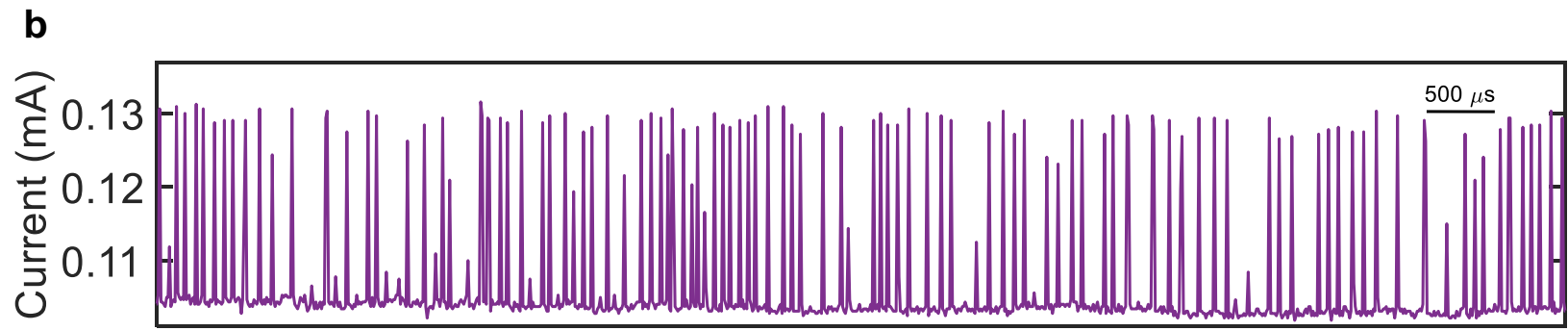

Time

Figure S4: Examples of spiking activity $\left(t_{U}<<t_{D}\right)$ measured with $10 \mu$ s sampling interval. Spiking is due to repeated switching events at a single site in the network. (a) Segment of data from Device 1 showing homogeneous spiking activity characterized by spikes with very similar amplitude. Each panel shows data at 10 times shorter time scale than the previous one. (b) Segment of data from Device 1 showing unresolved spikes with smaller amplitude occurring faster than the sampling interval $(10 \mu \mathrm{s})$. Unresolved spikes are much less common at sampling interval of $1 \mu \mathrm{s}$ as shown in Figure S3. 

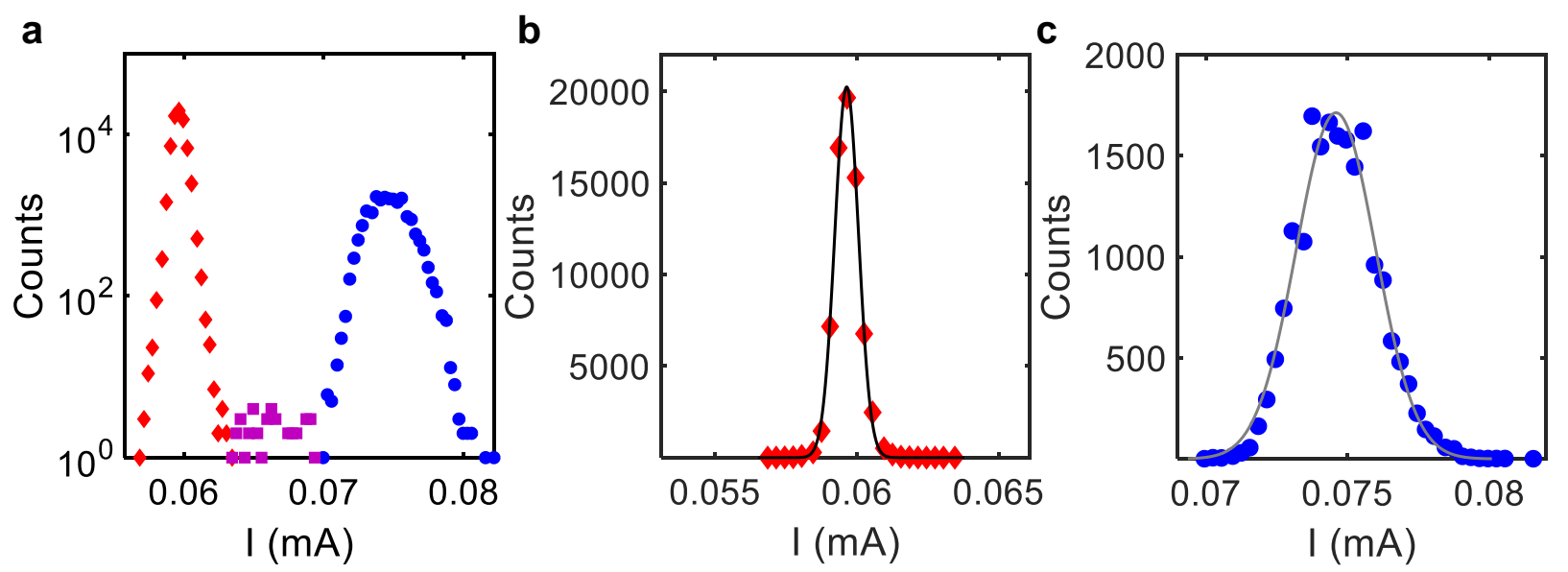

Figure S5: (a) Histograms of the measured current from a section of the data from Device 1 shown in Figure 2a. The currents in the DOWN and UP states are shown in red (diamonds) and blue (circles), respectively. The magenta squares correspond to the few measured currents between the DOWN and UP states. Note the logarithmic vertical scale: the two peaks have approximately normal distributions, as shown in panels b and c. (b) Distribution of currents measured while the site is in the DOWN state. (c) Distribution of currents measured while the site is in the UP state. Least squares regression lines for the normal distributions are shown in black and gray. The full width at half maximum (FWHM) of the distribution of the UP state is approximately five times larger than the one of the DOWN state $\left(F W H M_{D O W N}=0.001 \mathrm{~mA}, F W H M_{U P}=0.005 \mathrm{~mA}\right)$. In the DOWN state the atomic filament within the spiking tunnel gap is absent and therefore, the width of the normal distribution corresponds to the noise from the measurement instrumentation. The larger width of the normal distribution for the UP state is a direct consequence of atomic-scale variations in the structure of the atomic filament within the spiking tunnel gap. ${ }^{5}$ 


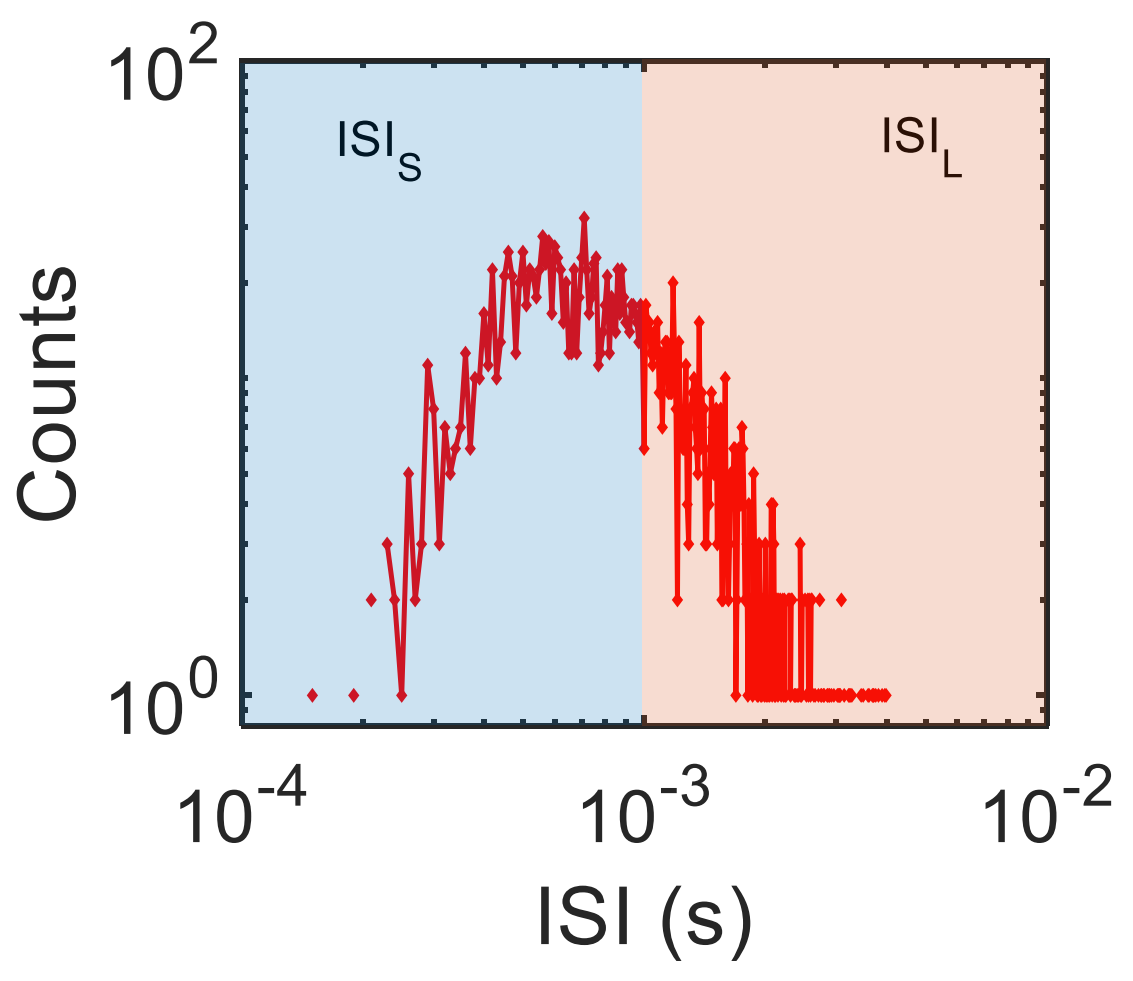

Figure S6: Histogram of the inter-spike intervals (ISIs) for the dataset presented in Figure 3b. Short and long ISIs (ISI $\mathrm{S}_{\mathrm{S}}$ and $\mathrm{ISI}_{\mathrm{L}}$ ) are highlighted by the blue and orange shaded areas, respectively. The line separating the two areas is the mean ISI. Note that because of the long tail of the distribution (small number of very large ISIs) the mean is greater than than the median. 

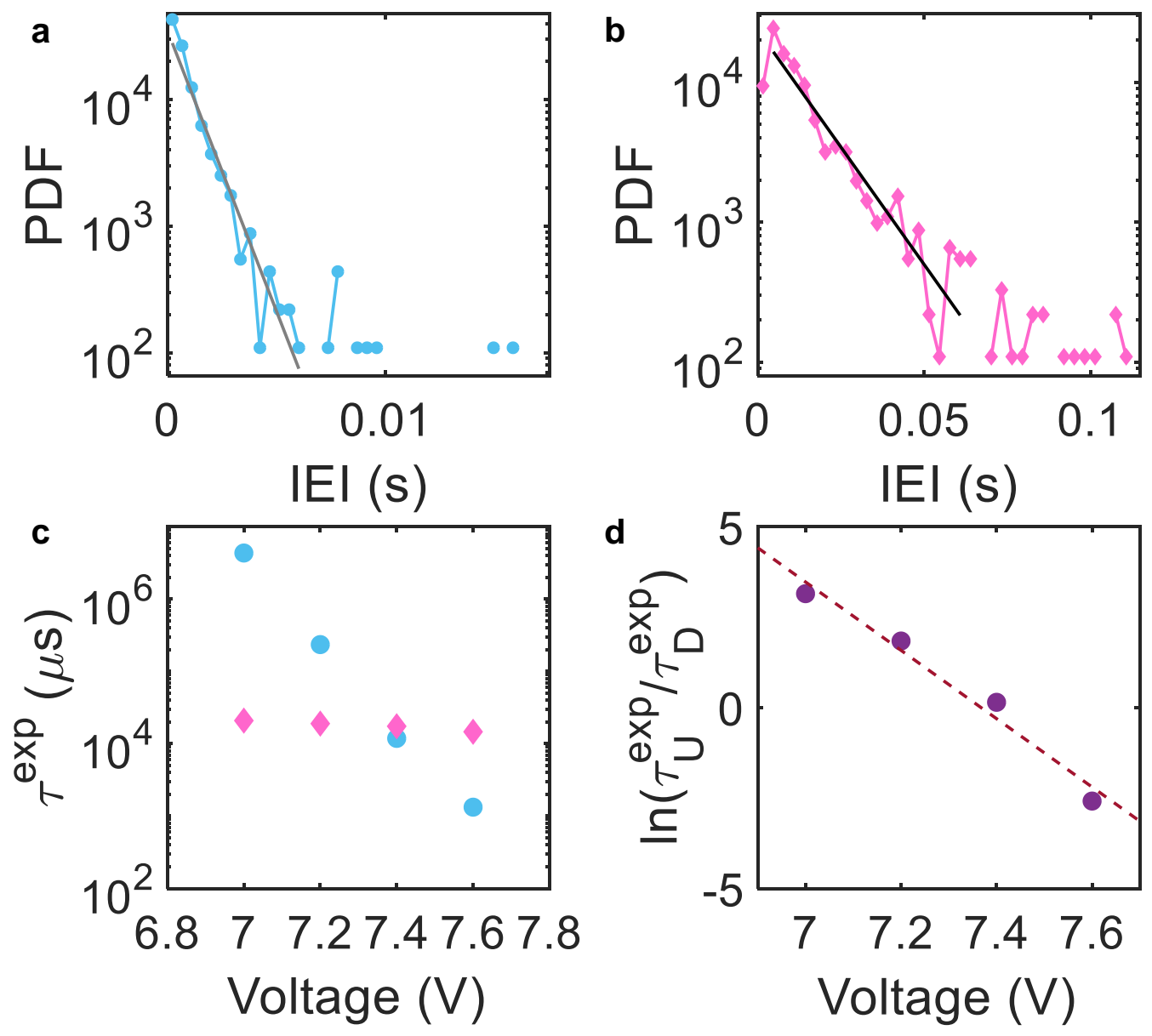

Figure S7: Analysis of data from Figure 4 of the main text using a standard methodology ${ }^{6}$ for random telegraph noise (RTN). This analysis assumes an exponential distribution of UP and DOWN times, $a e^{-t / \tau^{e x p}}$. (a-b) Histograms (linear bin sizes) of the inter-event intervals of Device 3 at an applied voltage of $7.6 \mathrm{~V}$. Note the semi-log scales (in contrast, Figure 4 is a log-log plot). Panel a shows $t_{U}$ while panel b shows $t_{D}$. Exponential maximum likelihood (ML) fits are presented in gray and black. Note that the head and tail of the distributions cannot be fitted by exponentials whereas the log-normal analysis presented in the main text achieves good fits over the entire range of IEIs. (c) Fitted parameters $\tau_{U}^{e x p}$ (cyan) and $\tau_{D}^{e x p}$ (pink) as a function of applied voltage. $\tau_{U}^{e x p}$ decreases with increasing applied voltage while $\tau_{D}^{e x p}$ remains constant. The qualitative behavior of $\tau_{U}^{e x p}$ and $\tau_{D}^{e x p}$ is the same as that found for the log-normal analysis described in Figure 4 but the physical meaning of the analysis is different - see main text. (d) Ratio between $\tau_{U}^{e x p}$ and $\tau_{D}^{e x p}$ as a function of applied voltage. 
Table S1: Summary of the analyzed sequences of data from three different devices. Each dataset corresponds to a different spike train (for example Figures 2c and e) originating from a different tunnel gap within the device. See main text for discussion. The first column identifies the dataset under analysis. The next six columns contain the log-normal ML estimators $\mu$ and $\sigma$ and the mean time in each state $\tau$ calculated as $\tau=e^{\mu+\frac{1}{2} \sigma^{2}}$ (Ref. 7) for both UP and DOWN states. $\sigma$, which is related to the variance of the log-normal distributions, does not vary significantly between UP and DOWN states. Moreover, $\sigma$ values are within an order of magnitude for all datasets and for all devices. $\tau_{D}$ is comparable for all datasets from each device but $\tau_{U}$ changes significantly. $\tau_{U} / \tau_{D}$ is the ratio between the mean time in the UP state and that in the DOWN state. Columns $\tau_{U}^{e x p}$ and $\tau_{D}^{\text {exp }}$ report the time constants obtained from the exponential ML estimators. The fitted parameters for the exponential fits follow the same trends as observed in the log-normal ones. $\tau_{U}^{\text {exp }} / \tau_{D}^{\text {exp }}$ is the ratio between the exponential time constants for UP and DOWN times, respectively. $d I_{\text {peak }}$ is the peak of the histogram of the changes in current i.e. the difference in current between the UP and DOWN states. $V_{a p p}$ is the applied DC voltage during the measurement. The last column shows the rate of events.

\begin{tabular}{|c|c|c|c|c|c|c|c|c|c|c|c|c|c|}
\hline Dataset & $\mu_{U}$ & $\sigma_{U}$ & $\begin{array}{c}\boldsymbol{\tau}_{U} \\
{[\mu \mathrm{s}]}\end{array}$ & $\mu_{D}$ & $\sigma_{D}$ & $\begin{array}{c}\tau_{D} \\
{[\mu s]}\end{array}$ & $\frac{\tau_{U}}{\tau_{D}}$ & $\begin{array}{l}\tau_{U}^{\exp } \\
{[\mu \mathrm{s}]}\end{array}$ & $\begin{array}{l}\tau_{D}^{\exp } \\
{[\mu \mathrm{s}]}\end{array}$ & $\frac{\tau_{U}^{\exp }}{\tau_{D}^{\exp }}$ & $\begin{array}{c}d I_{\text {peak }} \\
{[\mu \mathrm{A}]}\end{array}$ & $\begin{array}{c}V_{a p p} \\
\text { [V] }\end{array}$ & $\begin{array}{c}\text { Rate } \\
\text { [Count/s] }\end{array}$ \\
\hline \multicolumn{14}{|c|}{ Device 1} \\
\hline 1 & 0.53 & 0.42 & 19 & 4.46 & 0.52 & 988 & 0.02 & 4 & 718 & 0.01 & 24 & 8.0 & 1970 \\
\hline 2 & 0.42 & 0.39 & 16 & 4.28 & 0.47 & 809 & 0.02 & 5 & 430 & 0.01 & 23 & 8.0 & 2240 \\
\hline 3 & 0.01 & 0.64 & 12 & 2.01 & 0.54 & 85 & 0.14 & 12 & 50 & 0.24 & 25 & 8.0 & 20000 \\
\hline 4 & 0.19 & 0.37 & 13 & 1.92 & 0.41 & 75 & 0.17 & 4 & 36 & 0.12 & 21 & 8.0 & 22900 \\
\hline 5 & 2.68 & 0.82 & 205 & 3.05 & 0.66 & 260 & 0.79 & 200 & 200 & 1.00 & 19 & 8.0 & 4160 \\
\hline 6 & 3.65 & 0.67 & 483 & 5.09 & 0.55 & 1880 & 0.26 & 400 & 970 & 0.41 & 16 & 8.0 & 802 \\
\hline 7 & 2.43 & 1.05 & 198 & 3.90 & 0.54 & 573 & 0.35 & 190 & 430 & 0.44 & 23 & 8.0 & 2580 \\
\hline 8 & 1.42 & 0.58 & 49 & 2.77 & 0.52 & 182 & 0.27 & 26 & 100 & 0.26 & 16 & 8.0 & 8740 \\
\hline \multicolumn{14}{|c|}{ Device 2} \\
\hline 9 & 2.01 & 1.20 & 154 & 5.10 & 0.65 & 2010 & 0.08 & 200 & 1300 & 0.15 & 10 & 9.0 & 879 \\
\hline 10 & 1.30 & 1.10 & 67 & 7.28 & 0.63 & 17700 & 0.00 & 120 & 14000 & 0.01 & 30 & 8.0 & 113 \\
\hline 11 & 1.73 & 1.70 & 242 & 7.48 & 0.39 & 19100 & 0.01 & 200 & 7700 & 0.03 & 20 & 9.0 & 120 \\
\hline 12 & 1.76 & 1.10 & 106 & 6.84 & 0.82 & 13200 & 0.01 & 100 & 8100 & 0.01 & 65 & 5.0 & 143 \\
\hline 13 & 3.14 & 0.83 & 328 & 6.05 & 0.45 & 4690 & 0.07 & 400 & 1300 & 0.31 & 17 & 10.0 & 401 \\
\hline 14 & 5.97 & 1.48 & 11732 & 6.80 & 0.49 & 10100 & 1.16 & 20000 & 2900 & 6.90 & 7 & 10.0 & 100 \\
\hline 15 & 6.60 & 1.11 & 13570 & 8.15 & 0.42 & 37600 & 0.36 & 16200 & 13000 & 1.25 & 11 & 10.0 & 45 \\
\hline 16 & 5.50 & 1.56 & 8251 & 7.02 & 0.32 & 11800 & 0.70 & 7600 & 3930 & 1.93 & 17 & 10.0 & 100 \\
\hline \multicolumn{14}{|c|}{ Device 3} \\
\hline 17 & 0.75 & 0.56 & 25 & 5.02 & 0.68 & 1900 & 0.01 & 14 & 1400 & 0.01 & 15 & 8.0 & 1030 \\
\hline 18 & 1.25 & 0.73 & 46 & 5.33 & 0.72 & 2670 & 0.02 & 33 & 2100 & 0.02 & 14 & 8.0 & 665 \\
\hline 19 & 2.01 & 1.25 & 162 & 5.65 & 0.84 & 4050 & 0.04 & 134 & 3500 & 0.04 & 11 & 8.0 & 415 \\
\hline 20 & 3.18 & 1.62 & 903 & 5.30 & 0.80 & 2760 & 0.33 & 693 & 2000 & 0.35 & 15 & 8.0 & 547 \\
\hline 21 & 1.68 & 1.06 & 94 & 3.96 & 1.48 & 1560 & 0.06 & 136 & 2400 & 0.06 & 27 & 8.0 & 1720 \\
\hline 22 & 1.30 & 1.59 & 133 & 2.50 & 1.24 & 263 & 0.51 & 346 & 336 & 1.03 & 27 & 8.0 & 4040 \\
\hline 23 & 3.20 & 1.57 & 865 & 4.00 & 1.23 & 1150 & 0.75 & 946 & 923 & 1.02 & 32 & 8.0 & 1100 \\
\hline 24 & 0.09 & 1.70 & 46 & 2.50 & 1.34 & 299 & 0.16 & 167 & 438 & 0.38 & 29 & 8.0 & 3700 \\
\hline
\end{tabular}



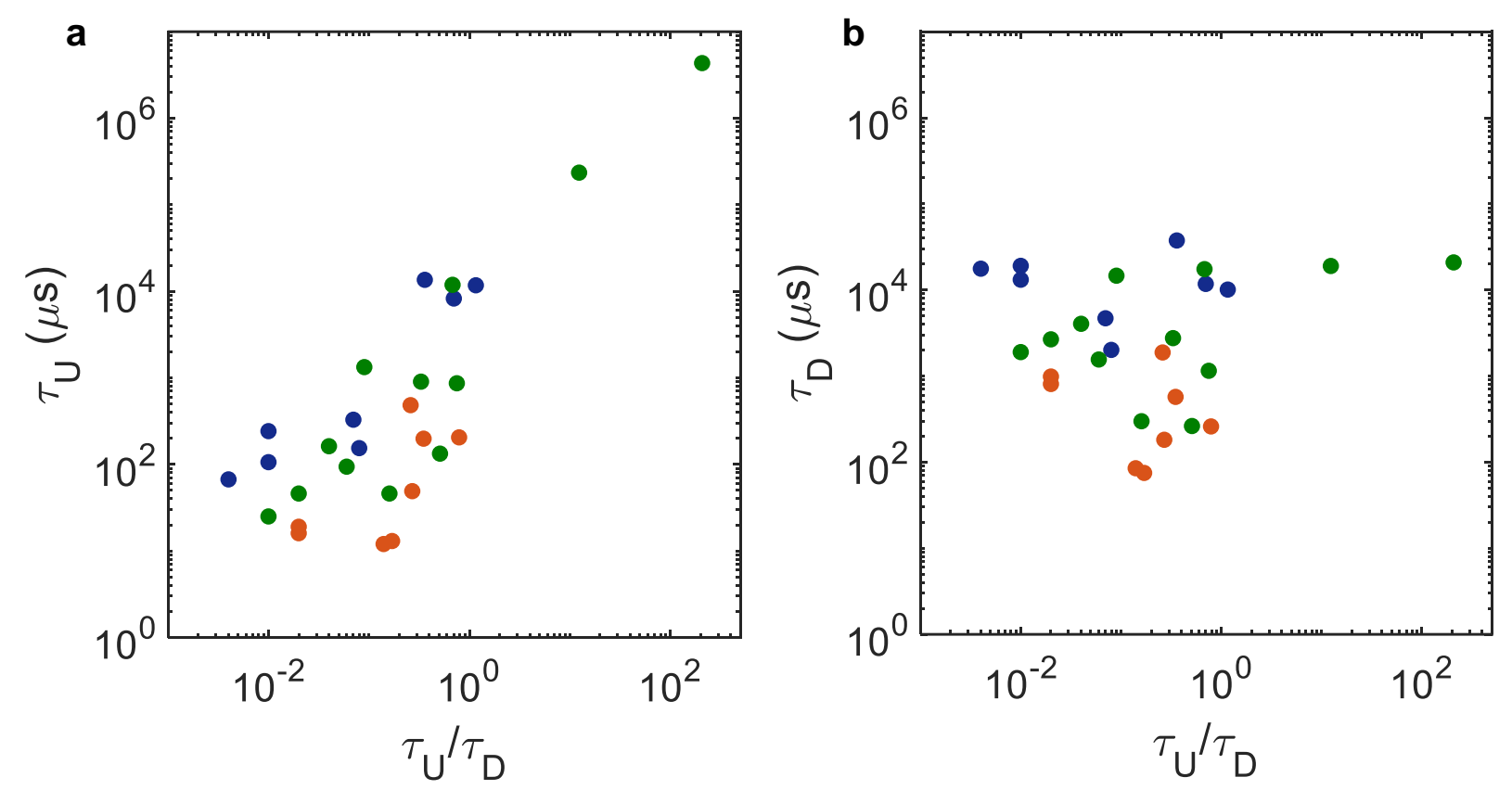

Figure S8: Variation of the mean times in the UP and DOWN states. $\tau_{U}$ (panel a) and $\tau_{D}$ (panel b) are plotted against the ratio $\tau_{U} / \tau_{D}$ for all the spiking data shown in Table S1. Each point represents a different segment of data similar to those shown in Figures S3 and S4. Blue: Device 1, orange: Device 2, green: Device 3. While there is scatter in the data, and some variation from sample to sample, $\tau_{U}$ increases approximately linearly with $\tau_{U} / \tau_{D}$ while $\tau_{D}$ is independent of $\tau_{U} / \tau_{D}$. Hence the time required to break a filament varies significantly from device-to-device and example-to-example but the time to form a filament is much less variable - see discussion in Mechanisms section of the main text. 

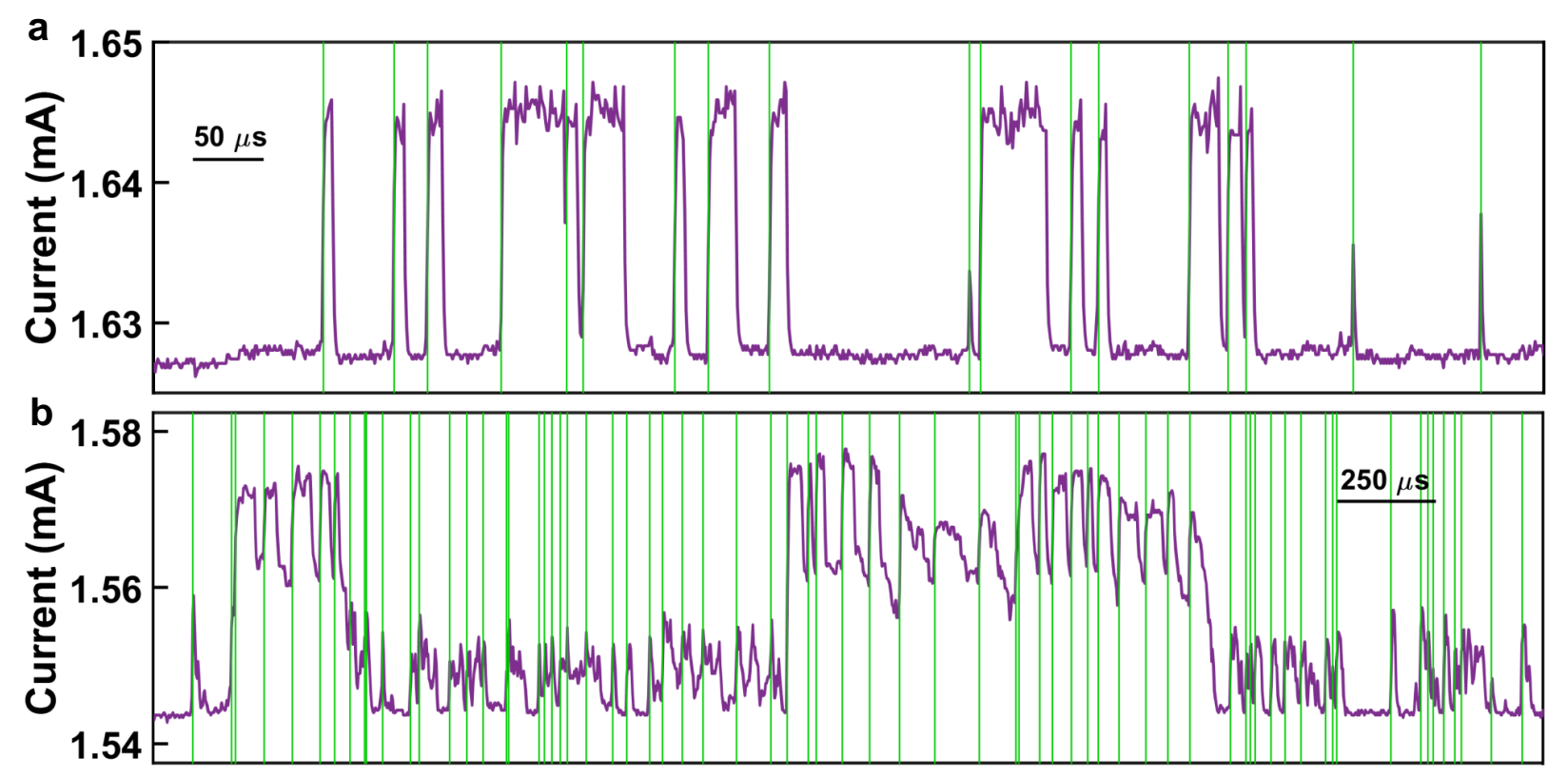

Time

Figure S9: Examples of detected rising edges of spikes using the TRNG circuit, showing robustness of the method. (a) Example of two-level homogeneous spiking activity measured from Device 2 with a sampling interval of $1 \mu \mathrm{s}$. The detected rising edges are highlighted in green and are used for the random number generation as explained in the main text. (b) Example of less pure spiking activity measured from Device 2 with a sampling interval of $1 \mu \mathrm{s}$. 


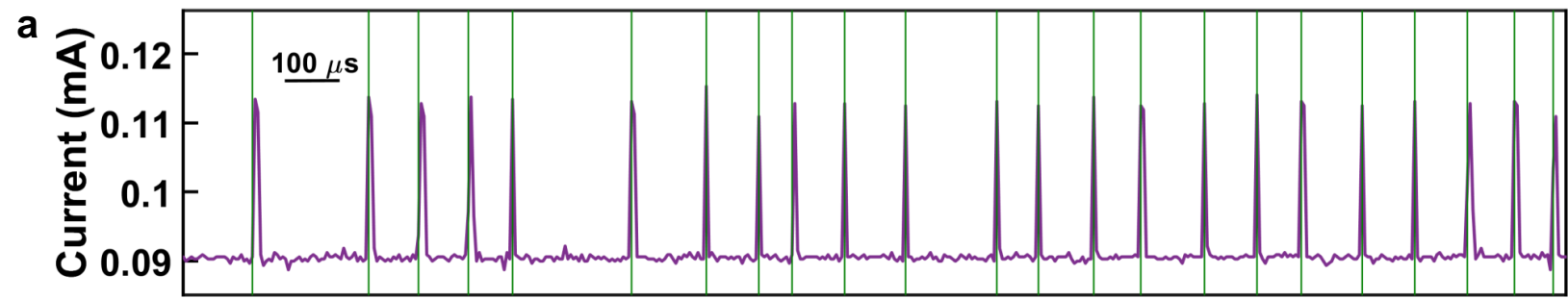

Time

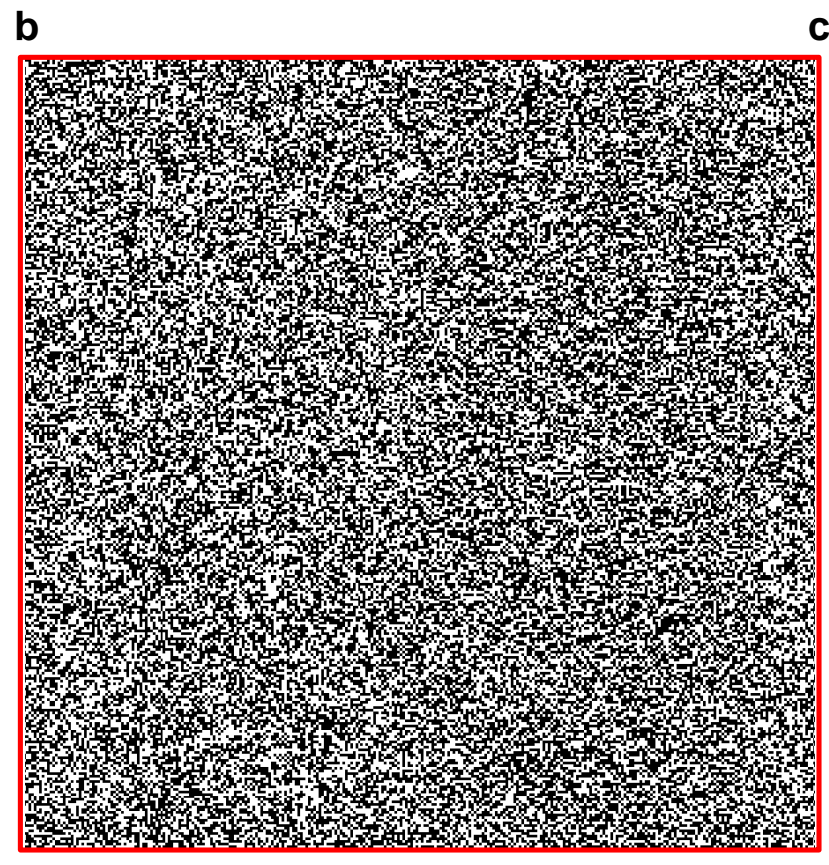

C

\begin{tabular}{|l|c|c|}
\hline Device 1 & $\begin{array}{l}\text { NIST STATISTICAL TEST SUITE } \\
\text { SP 800-22 }\end{array}$ \\
\hline Test & P-value & Pass-Rate \\
\hline Frequency & 0.0089 & $98 / 100$ \\
\hline Block Frequency & 0.0001 & $100 / 100$ \\
\hline Cumulative & 0.3505 & $98 / 100$ \\
Sums & 0.1453 & $96 / 100$ \\
\hline Runs & 0.7792 & $99 / 100$ \\
\hline Longest Run & 0.5141 & $99 / 100$ \\
\hline Rank & 0.9643 & $19 / 20$ \\
\hline FFT & 0.0156 & $100 / 100$ \\
\hline Non-Overlapping & $>0.0058$ & $145 / 148$ \\
\hline Template & 0.0002 & $97 / 100$ \\
\hline $\begin{array}{l}\text { Overlapping } \\
\text { Template }\end{array}$ & 0.7316 & $2 / 2$ \\
\hline Universal & 0.3191 & $98 / 100$ \\
\hline Approximate \\
Entropy
\end{tabular}

Figure S10: True Random Number Generation from data measured with a sampling interval of $10 \mu \mathrm{s}$. (a) Example of two-level homogeneous spiking activity measured from Device 1. The detected rising edges are highlighted in green and are used for the random number generation as explained in the main text. (b) Visual representation of a portion of a bit stream generated using data from Device 1. Each pixel represents a bit. White: ones, black: zeroes. (c) Results obtained from the NIST Statistical Test Suite SP 800-22. ${ }^{8}$ The analyzed spiking sequence generated 800 kbits $(\mathrm{m}=4 \mathrm{LSBs}) .12$ out of 15 NIST tests were applied following the protocols suggested by NIST, see methods for details. In particular, all tests were run on 100 sequences of 8000 bits, except the rank test which was run on 20 sequences of 39000 bits and the universal test which was run on 2 sequences of 400000 bits. Each test is considered passed and the bit-stream is considered indistinguishable from a random one if the pass rate is at least 96/100 and if p-value $>0.0001$. Due to the limited number of sequences, the $\mathrm{p}$-value reported for the universal test is the average of the $\mathrm{p}$-values found for each sequence. The generated bit-stream passes all the applied tests without post-processing. 

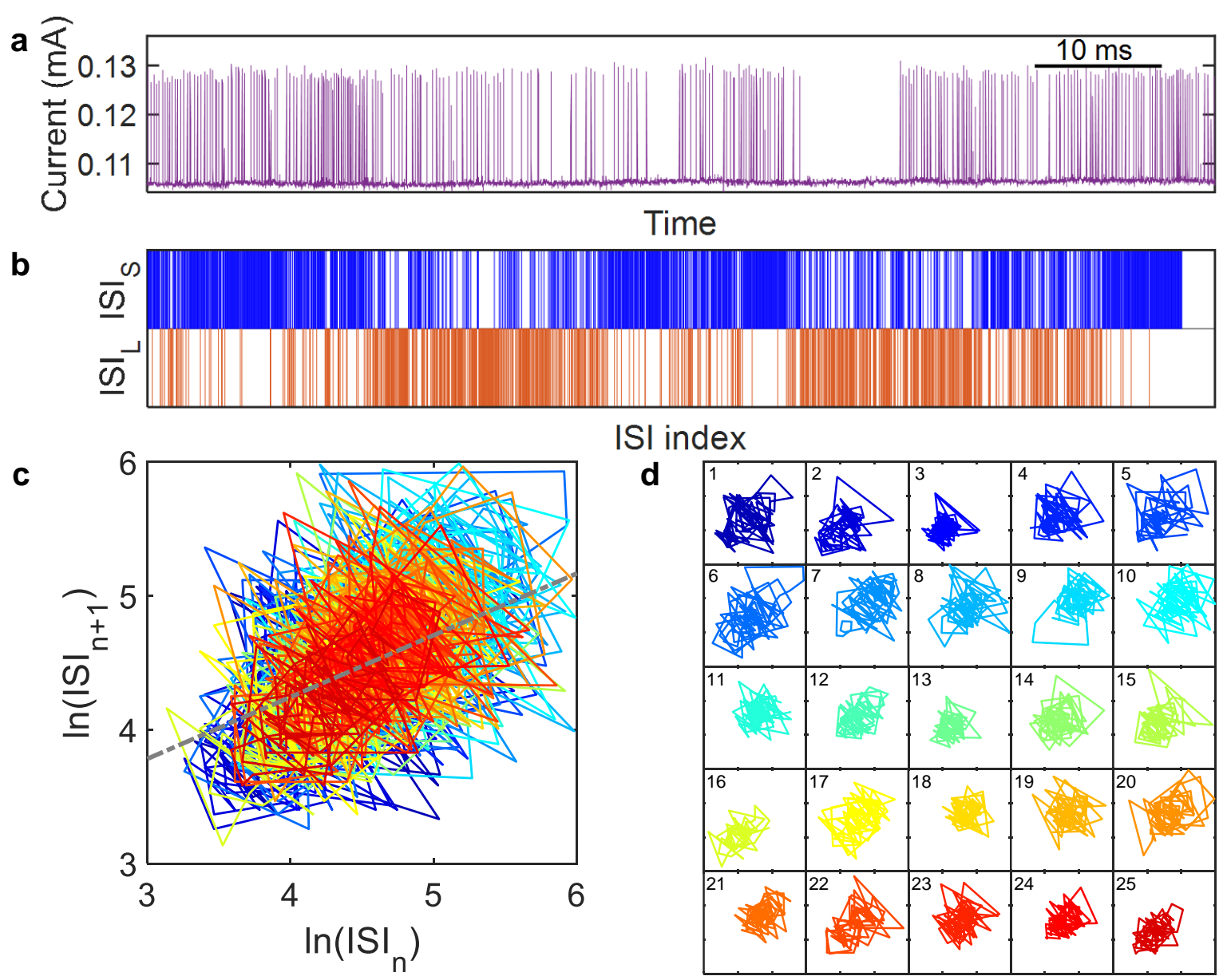

Figure S11: Stochasticity and serial correlations. Reproduction for the reader's convenience of Figure 3 from the main text. 


\section{Supplementary Section II: Serial Correlation}

Serial correlation in a sequence of inter-spike-intervals (ISIs) ${ }^{1}$ is commonly observed for biological neurons, ${ }^{9-11}$ i.e. successive ISIs are dependent on each other. These correlations are important in neural systems because they are associated with firing-rate stabilization, improved detectability of weak sensory signals, and enhanced transmission of information. ${ }^{12}$ Negative serial correlation means that a short ISI has the tendency to be followed by a long ISI and vice versa. Mechanisms include synaptic depression, delayed feedback, threshold fatigue and cumulative growth in spikes after hyperpolarization. ${ }^{12,13}$ Positive serial correlation indicates that (on average) long ISIs follow long ISIs and short ISIs are followed by short ISIs. Positive serial correlation has been experimentally observed in, for example, sensory neurons ${ }^{11}$ and afferent neurons in Zebrafish. ${ }^{10}$ At a mechanistic level, such correlations originate from phenomena such as intrinsic noise from ion channels with slow kinetics, ${ }^{11}$ fluctuations in total calcium channel activity or multiple heterogeneous synaptic contacts, ${ }^{10}$ slow external stimulus ${ }^{9}$ and stochastic adaption. ${ }^{14}$ In biological neurons, positive serial correlation facilitates certain classes of neuronal coding. ${ }^{12}$ For example, in motor systems, where rate coding is believed to be more important than temporal coding, reduced variability in ISIs helps produce smooth, accurate movements. ${ }^{15}$ Recent theoretical work suggests that positive serial correlations maximize information transmission at certain frequencies. ${ }^{16}$

Positive serial correlation - clustering of short and long ISIs - is observed in spike trains from our devices, as shown in Figure S11a. This indicates that the 'neurons' in our PNNs switch between slower and faster modes of spike generation, as in biological neurons. ${ }^{10} \mathrm{We}$ speculate that the positive serial correlation in the ISIs of our devices results from a combination of two physical processes operating in different dynamical regimes. The filament formation/rupture processes (corresponding to spiking) occur in a single tunnel-gap and are fast. Meanwhile, the growth/relaxation of 'hillocks' (early stages of filament formation, orange atoms in Figure 5 main text) within other tunnel-gaps throughout the network are

\footnotetext{
${ }^{1}$ Note that an inter-spike-interval corresponds to the sum of the up and down times discussed in the main text.
} 
responses to lower electric fields and so are relatively slow. Small scale, slow fluctuations in hillock sizes in the other tunnel gaps will modulate ${ }^{3}$ the potential across the spiking junction, altering the spiking rate. This argument is supported by the observation (Figure 4c in the main text) that a very small change in applied potential can lead to drastic changes in spiking rate. In summary: the slow changes in potential caused by hillock growth/relaxation modulate the much faster spiking events, leading to the observed positive serial correlation.

\section{Supplementary Section III: Comparison of TRNG performance}

In this section we compare the performance of the PNN-TRNG reported in Figure 6 of the main text with other TRNGs reported in the literature. ${ }^{17-32}$

Table S2 compares performance according to four key metrics: bit rate, whether postprocessing is required, number of bits that could be tested, and performance in standard benchmark tests (NIST Statistical Test Suite ${ }^{8}$ ). Also indicated are the underlying device technology and the method used to generate the bit stream. The methods can be divided into two broad categories i.e. conversion of measured times and conversion of measured levels (i.e. output currents, voltages, etc.) into random bits.

The color-coding in Table S2 provides a qualitative indication of performance according to each metric. The bit rate of the PNN-TRNG is competitive with, and often exceeds, that of the other TRNGs reported. As mentioned in the main text, the bit rate could be improved further by using standard techniques such as multi-bit linear feedback shift registers (LFSRs), which would allow achievement of bit rates over 100 Mbits s $^{-1}$ as reported in Refs. 17,29. Note that 1) the PNN-TRNG achieves raw bit rates around three orders of magnitude larger than the raw bit rates (i.e. prior to the LFSR) of Refs. 31,32. 2) In most reports in the literature it was not possible to obtain the very long bit streams required to run all NIST tests. 3) In several studies at least one of the reported NIST tests appears to have been run on sequences which do not comply with NIST recommendations ${ }^{8}$ (e.g. number of bits appears insufficient for the test). Table S2 emphasizes that our TRNG shows competitive 
performance for all the different metrics without the use of post-processing techniques.

Finally, we note that an ideal TRNG requires low power-consumption and small area. Typical power consumption in our devices is $\sim 10 \mathrm{~mW}$ which corresponds to $\sim 10^{-7} \mathrm{~J} \mathrm{bit}^{-1}$. The present devices have areas of $\sim 10^{-1} \mathrm{~mm}^{2}$ but, as noted in the main text, the spiking behavior that is utilized for random number generation is from a single nanoscale tunnel junction within the device. Hence there are significant opportunities for miniaturization of the devices. A detailed comparison of these metrics with other devices is difficult these data are often not reported in the literature. We note that Refs. 23 and 28 report very low power consumption (see footnotes in Table S2), but performance is less strong in other metrics. 
Table S2: Comparison of TRNG performance with results from the literature. The first three columns identify, respectively, the references from which the data in the table was extracted, the underlying device technology, and the method used to generate the random numbers. The table contains two rows for TRNGs where results were reported both with and without post-processing. The four color-coded columns report key performance metrics for each TRNG: the obtained bit-rate, whether post-processing is required, the number of bits tested, and reported benchmark results from the NIST Statistical Test Suite. ${ }^{8}$ The colorcoding is used to give a qualitative indication of TRNG performance. The $*$ indicates that at least one of the reported NIST tests appears to have been run on sequences which do not comply with NIST recommendations ${ }^{8}$ (e.g. reported number of bits was not sufficient for the test).

\begin{tabular}{|c|c|c|c|c|c|c|}
\hline Ref. & Technology & Method & $\begin{array}{c}\text { Bit-rate } \\
\text { [kbits s }{ }^{-1} \text { ] }\end{array}$ & $\begin{array}{l}\text { Post- } \\
\text { processing }\end{array}$ & $\begin{array}{c}\text { Bits } \\
\text { tested }\end{array}$ & $\begin{array}{c}\text { Benchmark } \\
\text { NIST STS }\end{array}$ \\
\hline This work & PNN & Spiking Time & $\sim 30$ & No & $1.8 \mathrm{M}$ & $12 / 12$ \\
\hline Jiang et al. [17] & Diffusive Memristor & Switching Time & $\sim 6$ & No & $76 \mathrm{M}$ & $15 / 15$ \\
\hline Jerry et al. [18] & IMT Oscillator & Phase Transition Time & $\sim 60$ & No & $87 k$ & $12 / 12^{*}$ \\
\hline Woo et al. [19] & Two Coupled Memristors & Switching Time & $\sim 3$ & No & $300 \mathrm{k}$ & $8 / 8$ \\
\hline \multirow[b]{2}{*}{ Fukushima et al. [20] } & \multirow[b]{2}{*}{ p-MTJ } & \multirow[b]{2}{*}{ Switching Level } & $\sim 550^{\mathrm{a}}$ & No & $120 G^{b}$ & $0 / 15$ \\
\hline & & & & Cascaded XOR & $120 G^{b}$ & $15 / 15$ \\
\hline Balatti et al. [21] & RRAM & Switching Level & $\sim 200$ & No & NA & NA \\
\hline Gaviria-Rojas et al. [22] & SWCNT & Switching Level & NA & Von Neumann & $61 \mathrm{k}$ & $9 / 9$ \\
\hline \multirow{2}{*}{ Vodenicarevic et al. [23] $\#$} & \multirow{2}{*}{ SPMTJ } & \multirow{2}{*}{ Switching Level } & $\sim 5$ & No & $\sim 1 \mathrm{G}^{\mathrm{c}}$ & $0 / 15$ \\
\hline & & & $\sim 0.6^{\mathrm{d}}$ & Cascaded XOR & $\sim 130 \mathrm{M}^{e}$ & $15 / 15$ \\
\hline Carboni et al. [24] & Differential Read Scheme MTJ & Switching Level & $\sim 500$ & No & $1 \mathrm{M}$ & $10 / 10$ \\
\hline Chai et al. [25] & OTS & Switching Level & NA & No & $10 \mathrm{k}$ & $12 / 12^{*}$ \\
\hline Balatti et al. [26] & Two Coupled RRAM & Switching Level Differences & $\sim 0.16$ & Von Neumann & $2.2 \mathrm{M}$ & $9 / 9$ \\
\hline Zhang et al. [27] & Differential Two Memristors & Switching Level Differences & $\sim 0.01$ & Von Neumann & $23 \mathrm{k}$ & $12 / 12^{*}$ \\
\hline \multirow{2}{*}{ Aziza et al. [28] ${ }^{+}$} & \multirow{2}{*}{ ReRAM Array } & \multirow{2}{*}{ Switching Level Differences } & NA & No & $49 \mathrm{k}$ & $6 / 15^{\star}$ \\
\hline & & & NA & Cascaded XOR & $1 \mathrm{k}^{\dagger}$ & $12 / 15^{*}$ \\
\hline Huang et al. [29] & CRRAM & RTN Level & $\sim 1$ & No & NA & $5 / 5$ \\
\hline Brown et al. [30] & CMOS Transistor & RTN Level & 19.2 & No & $3 \mathrm{M}$ & $15 / 15^{\star}$ \\
\hline \multirow[b]{2}{*}{ Li et al. [31] } & \multirow[b]{2}{*}{ MIM } & \multirow[b]{2}{*}{ RTN Level } & $\sim 0.01$ & No & NA & $11 / 11^{*}$ \\
\hline & & & $\sim 1000$ & NLFSR & $30 \mathrm{M}$ & $15 / 15$ \\
\hline \multirow{2}{*}{ Wen et al. [32] } & \multirow{2}{*}{ MIM } & \multirow{2}{*}{ RTN Level } & $\sim 0.01$ & No & NA & $11 / 11^{*}$ \\
\hline & & & $\sim 1000$ & NLFSR & $18 \mathrm{G}$ & $15 / 15$ \\
\hline
\end{tabular}

\footnotetext{
a,b It is not clear whether these figures are for raw or post-processed bitstreams. ${ }^{\mathrm{c}}$ Estimated by dividing the raw sequence length of 21.2 Gbits by a factor of 20 which corresponds to the ratio between the raw sampling frequency $(100 \mathrm{kHz})$ and the subsampled working frequency $(5 \mathrm{kHz})$. ${ }^{\mathrm{d}}$ Estimated by dividing the raw bit rate of $5 \mathrm{kbits} \mathrm{s}^{-1}$ by a factor of 8 to account for the XOR 8 post-processing procedure. ${ }^{e}$ Estimated by dividing the sequence length of 1 Gbits by a factor of 8 to account for the XOR8 post-processing procedure. ${ }^{\mathrm{f}}$ Estimated by dividing the raw sequence length of 49 kbits by a factor of 49 to account for the XOR49 post-processing procedure. \# Estimated energy consumption $\sim 3 \mathrm{pJ} \mathrm{bit}^{-1}$. ${ }^{+}$Estimated energy consumption $\sim 138 \mathrm{pJ} \mathrm{bit}^{-1}$.
} 


\section{References}

(1) Mallinson, J. B.; Shirai, S.; Acharya, S. K.; Bose, S. K.; Galli, E.; Brown, S. A. Avalanches and Criticality in Self-Organized Nanoscale Networks. Science Advances 2019, 5, eaaw8438.

(2) Shirai, S.; Acharya, S. K.; Bose, S. K.; Mallinson, J. B.; Galli, E.; Pike, M. D.; Arnold, M. D.; Brown, S. A. Long-Range Temporal Correlations in Scale-Free Neuromorphic Networks. Network Neuroscience 2020, 4, 432-447.

(3) Pike, M. D.; Bose, S. K.; Mallinson, J. B.; Acharya, S. K.; Shirai, S.; Galli, E.; Weddell, S. J.; Bones, P. J.; Arnold, M. D.; Brown, S. A. Atomic Scale Dynamics Drive Brain-like Avalanches in Percolating Nanostructured Networks. Nano Letters 2020, 20, $3935-3942$.

(4) Friedman, N.; Ito, S.; Brinkman, B. A. W.; Shimono, M.; DeVille, R. E. L.; Dahmen, K. A.; Beggs, J. M.; Butler, T. C. Universal Critical Dynamics in High Resolution Neuronal Avalanche Data. Physical Review Letters 2012, 108, 208102.

(5) Wang, W.; Covi, E.; Lin, Y.-H.; Ambrosi, E.; Ielmini, D. Modeling of Switching Speed and Retention Time in Volatile Resistive Switching Memory by Ionic Drift and Diffusion. 2019 IEEE International Electron Devices Meeting (IEDM). 2019; pp 32.3.132.3.4.

(6) Soni, R.; Meuffels, P.; Petraru, A.; Weides, M.; Kügeler, C.; Waser, R.; Kohlstedt, H. Probing Cu Doped Ge0.3Se0.7 Based Resistance Switching Memory Devices with Random Telegraph Noise. Journal of Applied Physics 2010, 107, 024517.

(7) Crow, E. L.; Shimizu, K. Lognormal Distributions; Marcel Dekker New York, 1987.

(8) Rukhin, A.; Soto, J.; Nechvatal, J.; Smid, M.; Leigh, S.; Levenson, M.; Vangel, M.; Banks, D.; Heckert, A.; Dray, J.; Vo, S.; Bassham, L. A Statistical Test Suite for Ran- 
dom and Pseudorandom Number Generators for Cryptographic Applications; Special Publication (NIST SP 800-22 Rev. 1a), National Institute of Standards and Technology, Gaithersburg, MD, 2010.

(9) Lindner, B. Interspike Interval Statistics of Neurons Driven by Colored Noise. Physical Review E 2004, 69, 022901.

(10) Song, S.; Lee, J. A.; Kiselev, I.; Iyengar, V.; Trapani, J. G.; Tania, N. Mathematical Modeling and Analyses of Interspike-Intervals of Spontaneous Activity in Afferent Neurons of the Zebrafish Lateral Line. Scientific Reports 2018, 8, 14851.

(11) Fisch, K.; Schwalger, T.; Lindner, B.; Herz, A. V.; Benda, J. Channel Noise from Both Slow Adaptation Currents and Fast Currents is Required to Explain Spike-Response Variability in a Sensory Neuron. Journal of Neuroscience 2012, 32, 17332-17344.

(12) Avila-Akerberg, O.; Chacron, M. J. Nonrenewal Spike Train Statistics: Causes and Functional Consequences on Neural Coding. Experimental Brain Research 2011, 210, $353-371$.

(13) Farkhooi, F.; Strube-Bloss, M. F.; Nawrot, M. P. Serial Correlation in Neural Spike Trains: Experimental Evidence, Stochastic Modeling, and Single Neuron Variability. Physical Review E 2009, 79, 021905.

(14) Schwalger, T.; Fisch, K.; Benda, J.; Lindner, B. How Noisy Adaptation of Neurons Shapes Interspike Interval Histograms and Correlations. PLoS Computational Biology 2010, 6, e1001026.

(15) Stein, R. B.; Gossen, E. R.; Jones, K. E. Neuronal Variability: Noise or Part of the Signal? Nature Reviews Neuroscience 2005, 6, 389-397.

(16) Blankenburg, S.; Lindner, B. The Effect of Positive Interspike Interval Correlations on 
Neuronal Information Transmission. Mathematical Biosciences and Engineering 2016, 13, 461-481.

(17) Jiang, H.; Belkin, D.; Savel'ev, S. E.; Lin, S.; Wang, Z.; Li, Y.; Joshi, S.; Midya, R.; Li, C.; Rao, M.; Barnell, M.; Wu, Q.; Yang, J. J.; Xia, Q. A Novel True Random Number Generator Based on a Stochastic Diffusive Memristor. Nature Communications 2017, 8,882 .

(18) Jerry, M.; Ni, K.; Parihar, A.; Raychowdhury, A.; Datta, S. Stochastic Insulator-toMetal Phase Transition-Based True Random Number Generator. IEEE Electron Device Letters 2018, 39, 139-142.

(19) Woo, K. S.; Wang, Y.; Kim, J.; Kim, Y.; Kwon, Y. J.; Yoon, J. H.; Kim, W.; Hwang, C. S. A True Random Number Generator using Threshold-Switching-Based Memristors in an Efficient Circuit Design. Advanced Electronic Materials 2019, 5, 1800543.

(20) Fukushima, A.; Seki, T.; Yakushiji, K.; Kubota, H.; Imamura, H.; Yuasa, S.; Ando, K. Spin Dice: A Scalable Truly Random Number Generator Based on Spintronics. Applied Physics Express 2014, 7, 083001.

(21) Balatti, S.; Ambrogio, S.; Wang, Z.; Ielmini, D. True Random Number Generation by Variability of Resistive Switching in Oxide-Based Devices. IEEE Journal on Emerging and Selected Topics in Circuits and Systems 2015, 5, 214-221.

(22) Gaviria Rojas, W. A.; McMorrow, J. J.; Geier, M. L.; Tang, Q.; Kim, C. H.; Marks, T. J.; Hersam, M. C. Solution-Processed Carbon Nanotube True Random Number Generator. Nano Letters 2017, 17, 4976-4981.

(23) Vodenicarevic, D.; Locatelli, N.; Mizrahi, A.; Friedman, J. S.; Vincent, A. F.; Romera, M.; Fukushima, A.; Yakushiji, K.; Kubota, H.; Yuasa, S.; Tiwari, S.; Grol- 
lier, J.; Querlioz, D. Low-Energy Truly Random Number Generation with Superparamagnetic Tunnel Junctions for Unconventional Computing. Physical Review Applied 2017, 8, 054045 .

(24) Carboni, R.; Chen, W.; Siddik, M.; Harms, J.; Lyle, A.; Kula, W.; Sandhu, G.; Ielmini, D. Random Number Generation by Differential Read of Stochastic Switching in Spin-Transfer Torque Memory. IEEE Electron Device Letters 2018, 39, 951-954.

(25) Chai, Z.; Freitas, P.; Marsland, J.; Fantini, A.; Garbin, D.; Goux, L.; Kar, G. S.; Shao, W.; Zhang, W.; Brown, J.; Degraeve, R.; Salim, F. D.; Clima, S.; Hatem, F.; Zhang, J. F. GeSe-Based Ovonic Threshold Switching Volatile True Random Number Generator. IEEE Electron Device Letters 2020, 41, 228-231.

(26) Balatti, S.; Ambrogio, S.; Carboni, R.; Milo, V.; Wang, Z.; Calderoni, A.; Ramaswamy, N.; Ielmini, D. Physical Unbiased Generation of Random Numbers with Coupled Resistive Switching Devices. IEEE Transactions on Electron Devices 2016, 63, 2029-2035.

(27) Zhang, T.; Yin, M.; Xu, C.; Lu, X.; Sun, X.; Yang, Y.; Huang, R. High-Speed True Random Number Generation Based on Paired Memristors for Security Electronics. Nanotechnology 2017, 28, 455202.

(28) Aziza, H.; Postel-Pellerin, J.; Bazzi, H.; Canet, P.; Moreau, M.; Marca, V. D.; Harb, A. True Random Number Generator Integration in a Resistive RAM Memory Array Using Input Current Limitation. IEEE Transactions on Nanotechnology 2020, 19, 214-222.

(29) Huang, C.-y.; Shen, W. C.; Tseng, Y.-h.; King, Y.-c.; Lin, C.-j. A Contact-Resistive Random-Access-Memory-Based True Random Number Generator. IEEE Electron Device Letters 2012, 33, 1108-1110.

(30) Brown, J.; Zhang, J. F.; Zhou, B.; Mehedi, M.; Freitas, P.; Marsland, J.; Ji, Z. Random- 
Telegraph-Noise-Enabled True Random Number Generator For Hardware Security. Scientific Reports 2020, 10, 17210.

(31) Li, X.; Zanotti, T.; Wang, T.; Zhu, K.; Puglisi, F. M.; Lanza, M. Random Telegraph Noise in MetalOxide Memristors for True Random Number Generators: A Materials Study. Advanced Functional Materials 2021, 31, 2102172.

(32) Wen, C.; Li, X.; Zanotti, T.; Puglisi, F. M.; Shi, Y.; Saiz, F.; Antidormi, A.; Roche, S.; Zheng, W.; Liang, X.; Hu, J.; Duhm, S.; Roldan, J. B.; Wu, T.; Chen, V.; Pop, E.; Garrido, B.; Zhu, K.; Hui, F.; Lanza, M. Advanced Data Encryption using 2D Materials. Advanced Materials 2021, 33, 2100185. 